PROCEEDINGS OF THE

AMERICAN MATHEMATICAL SOCIETY

Volume 126, Number 2, February 1998, Pages 323-331

S 0002-9939(98)04446-3

\title{
IMAGE MULTIPLE POINT SPACES AND RECTIFIED HOMOTOPICAL DEPTH
}

\author{
KEVIN HOUSTON
}

(Communicated by Ron Donagi)

\begin{abstract}
We investigate the local topology of multiple point spaces in the image of a finite complex analytic map. In particular, we find examples of maps for which the constant sheaf on an image multiple point space of the map is perverse. These results are proved by the use of a spectral sequence which calculates the homology of the image of a continuous map.
\end{abstract}

\section{INTRODUCTION}

For a finite and proper complex analytic map $f: X \rightarrow Y$ the closure of the set of points in the image of $f$ that have $k$ or more preimages is a complex analytic space. These spaces are called the multiple point spaces in the image and are denoted $M_{k}(f)$.

In this paper we shall study the local topology of such spaces for certain classes of maps for which the image is a hypersurface in the target space. Here local topology means the study of punctured neighbourhoods of points. Knowledge of the behaviour of the local topology of a space can lead to information on the topology of the whole space, for instance, Lefschetz hyperplane theorems and Barth-Lefschetz type theorems; see for example [Ho2] and the references therein.

One of the consequences of the results is that we find examples of spaces where the sheaf complex defined by the constant sheaf $\mathbb{Q}_{X}$ in degree - $\operatorname{dim}_{\mathbb{C}} X$ and zero elsewhere, denoted $\mathbb{Q}_{X}^{\bullet}\left[\operatorname{dim}_{\mathbb{C}} X\right]$, is a perverse sheaf. The definition of a perverse sheaf can be found in, for example, $[\mathrm{M}]$.

In Section 1 we recall the definition of the multiple point spaces of a map and review rectified homotopical depth of a complex analytic space. When dealing with the topology of complex analytic spaces it is convenient to use the notion of rectified homotopical depth; this is essentially a measure of how the homotopy groups of punctured neighbourhoods of points vanish. The rational homological version of rectified homotopical depth is closely related to the perversity of the shifted constant sheaf.

The statements of the results on the rectified homotopical/homological depth of image multiple point spaces are made in Section 2 and the proofs are given in the following section. The final section contains some deductions from the theorems.

Received by the editors June 26, 1996.

1991 Mathematics Subject Classification. Primary 14B15, 32S05; Secondary 32S60.

Key words and phrases. Image computing spectral sequence, image multiple point space, perverse sheaf, singularity. 
Of particular interest is Corollary 2.9. From this we can deduce that if $f: X \rightarrow Y$ is a locally stable corank 1 map between complex manifolds of dimension $n$ and $n+1$ respectively, then the sheaf complex $\mathbb{Q}_{M_{k}(f)}^{\bullet}[n-k+1]$ is a perverse sheaf. Thus we have some new examples of perverse sheaves.

During the writing of this paper the author was supported by a grant from the EPSRC (grant number GR/K/29227).

\section{Notation AND DEFinitions}

Suppose $f: X \rightarrow Y$ is a finite and proper continuous map. Define the $k^{\text {th }}$ multiple point space $D^{k}(f)$ to be

$$
D^{k}(f):=\text { closure }\left\{\left(x_{1}, \ldots x_{k}\right) \in X^{k} \mid f\left(x_{1}\right)=\cdots=f\left(x_{k}\right) \text { for } x_{i} \neq x_{j}, i \neq j\right\} .
$$

Define the $k^{\text {th }}$ image multiple point space $M_{k}(f)$ to be

$$
M_{k}(f):=\operatorname{closure}\left\{y \in Y \mid \# f^{-1}(y)=k\right\} .
$$

There exist continuous maps $\varepsilon^{i, k}: D^{k}(f) \rightarrow D^{k-1}(f)$ defined by

$$
\varepsilon^{i, k}\left(x_{1}, \ldots, x_{k}\right)=\left(x_{1}, \ldots, \hat{x}_{i}, \ldots, x_{k}\right)
$$

for $1 \leq i \leq k$. We denote the image of $D^{j}(f)$ in $D^{k}(f)$ under any one of these maps by $D_{k}^{j}(f)$.

There also exist continuous maps $\varepsilon^{k}: D^{k}(f) \rightarrow Y$ given by

$$
\varepsilon^{k}\left(x_{1}, \ldots, x_{k}\right)=f\left(x_{i}\right) \text { for any } i .
$$

By definition we have $M_{k}(f)=\varepsilon^{k}\left(D^{k}(f)\right)=f\left(D_{1}^{k}\right)$.

Let $M$ be a module upon which $S_{k}$ acts (the group of permutations on $k$ objects), and give $S_{k}$ the usual sign representation. Then define the idempotent functor Alt to be given by

$$
\text { Alt } M=\left\{m \in M \mid \sigma(m)=\operatorname{sign}(\sigma) m \text { for all } \sigma \in S_{k}\right\} .
$$

One of the main theorems of $[\mathrm{GM}]$ concerns the existence of a spectral sequence calculating the rational homology of the image of $f$ :

Theorem 1.1. There exists a spectral sequence

$$
E_{1}^{p, q}(f)=\operatorname{AltH}^{q}\left(D^{p+1}(f) ; \mathbb{Q}\right) \Rightarrow H^{*}(f(X) ; \mathbb{Q}) .
$$

This sequence can be viewed as the multiple point spaces giving a resolution of the image. The behaviour of the multiple point spaces is often far simpler than that of the image. For example, if $f$ is a stable complex analytic map with the corank of its differential at every point less than or equal to 1 , then the multiple point spaces are nonsingular (see Theorem 2.14 of $[\mathrm{MM}]$ ), whereas, even in this case, in general the image multiple point spaces have non-isolated singularities.

Suppose $X$ is a complex analytic space. Let $\mathcal{S}$ be a Whitney stratification of $X$ and let $L_{A}$ denote the real link of the stratum $A$.

Definition 1.2. The rectified homotopical depth of $X$, denoted by $\operatorname{rhd}(X)$, is the largest integer such that

$$
\pi_{i}\left(\operatorname{Cone}\left(L_{A}\right), L_{A}\right)=0 \text { for } i<\operatorname{rhd}(X)-\operatorname{dim}_{\mathbb{C}} A \text { for all } A \in \mathcal{S} \text {. }
$$


This number does not depend on the stratification chosen. For the main theorems on rectified homotopical depth see [HL], in particular Theorems 2.12, 3.2.1 and 4.1.2.

We can make similar definitions for $r H d(X ; G)$, the rectified homological depth over $G$, by replacing the relative homotopy groups above by relative homology groups over the coefficient group $G$.

Suppose $X$ is an $S_{k}$-invariant complex analytic space with an $S_{k}$-invariant stratification, for example a multiple point space. Then we define rectified alternating homological depth, $\operatorname{raHd}(X ; \mathbb{Q})$, by choosing an $H$-invariant link of the stratum $A$, where $H$ is the isotropy subgroup of a point of $A$, and using the following:

$$
\operatorname{AltH}_{i}\left(\operatorname{Orbit}\left(\operatorname{Cone}\left(L_{A}\right)\right), \operatorname{Orbit}\left(L_{A}\right) ; \mathbb{Q}\right)=0 \text { for } i<\operatorname{raHd}(X)-\operatorname{dim}_{\mathbb{C}} A \text { for all } A \text {. }
$$

Examples of $\mathrm{raHd}$ are given in Theorem 2.13 of [Ho1] (where it was first introduced) and Proposition 3.4 of [Ho2].

In $[\mathrm{HL}]$ Hamm and Lê prove that the condition $r H d(X ; \mathbb{Q})=\operatorname{dim}_{\mathbb{C}} X$ is equivalent to $\mathbb{Q}_{X}^{\bullet}\left[\operatorname{dim}_{\mathbb{C}} X\right]$ being a perverse sheaf. They attribute this observation to Z. Mebkhout and an anonymous referee. The theorems in the next section give new examples of spaces for which the shifted constant sheaf is perverse.

\section{Statement of Results}

In this section we state the main results on the local topology of image multiple point spaces.

Definition 2.1. A complex analytic map $f: X \rightarrow Y$ is said to be induced from a codimension 1 map with property $A$ if there exists a finite and proper map $F: Z \rightarrow U$ such that $Z$ is a complex analytic space of dimension $n, U$ is a connected open subset of $\mathbb{C}^{n+1}, X=F^{-1}(Y)$ and $F$ has property $A$.

Example 2.2. If $f$ is a map of manifolds of dimension $n$ and $n+1$ and is finitely $\mathcal{A}$-determined, then $f$ is induced from a codimension one map which is stable. A good reference for notions from singularity theory, such as $\mathcal{A}$-determinacy, is the paper [W] by Wall.

Theorem 2.3. Suppose that $f: X \rightarrow Y$ is locally induced from codimension 1 maps of degree 1 with Gorenstein domains. Then

(i) $\operatorname{rhd}\left(M_{2}(f)\right) \geq \operatorname{rhd}(Y)-2$, and

(ii) $\operatorname{rhd}\left(D_{1}^{2}(f)\right) \geq \operatorname{rhd}(X)-1$.

The statement obtained by replacing rhd by $r H d$ is also true. In particular, supposing $f$ is dimensionally correct with $f(X)$ of codimension 1 in $Y$ and $r H d(Y ; \mathbb{Q})=$ $\operatorname{dim}_{\mathbb{C}} Y$, then $\mathbb{Q}_{M_{2}(f)}^{\bullet}\left[\operatorname{dim}_{\mathbb{C}} M_{2}(f)\right]$ is a perverse sheaf. Similarly, if $r H d(X ; \mathbb{Q})=$ $\operatorname{dim} X$, then $\mathbb{Q}_{D_{1}^{2}(f)}^{\bullet}\left[\operatorname{dim}_{\mathbb{C}} X-1\right]$ is perverse.

Thus assuming $X$ to be Gorenstein provides very good theorems on the local homotopy behaviour of $M_{2}(f)$ and $D_{1}^{2}(f)$. To prove theorems on other image multiple point spaces we move to homology and put stronger conditions on the spaces and maps. A suitable (generic) condition for a map is the following.

Definition 2.4. If $f: X \rightarrow Y$ is map of complex analytic spaces, then we say $f$ is dimensionally correct if $\operatorname{dim}_{\mathbb{C}} D^{k}(f)=k \cdot \operatorname{dim}_{\mathbb{C}} X-(k-1) \cdot \operatorname{dim}_{\mathbb{C}} Y$ for all $D^{k}(f) \neq \emptyset$.

That is, if $f$ is dimensionally correct, then the dimensions of the multiple point spaces are what one would expect from a generic map between manifolds. 
Example 2.5. Suppose $f$ is a finitely $\mathcal{A}$-determined map, $f: \mathbb{C}^{n} \rightarrow \mathbb{C}^{p}$, with $n<p$. Then it is proved in [Ho1] that $f$ is dimensionally correct.

Theorem 2.6. Suppose that $f: X \rightarrow Y$ is a dimensionally correct map, with $Y$ a complex analytic manifold of dimension $\operatorname{dim}_{\mathbb{C}} X+1=n+1$ and $r H d(X ; \mathbb{Q})=$ $\operatorname{dim}_{\mathbb{C}} X$. Then $r H d\left(M_{2} ; \mathbb{Q}\right) \geq \operatorname{rHd}\left(D_{1}^{2} ; \mathbb{Q}\right)$.

Thus, if $\mathbb{Q}_{X}^{\bullet}[n]$ and $\mathbb{Q}_{D_{1}^{2}}^{\bullet}[n-1]$ are perverse, then $\mathbb{Q}_{M_{2}}^{\bullet}[n-1]$ is perverse.

In the following a corank 1 map will be a map that at each point has differential of corank less than or equal to one. Corank 1 stable maps $f: X \rightarrow Y$ of complex manifolds of dimension $n$ and $n+1$ respectively are locally immersions or trivial extensions of maps of the form,

$$
\begin{aligned}
\left(x_{1}, \ldots, x_{2 k-3}, y\right) \mapsto & \left(x_{1}, \ldots, x_{2 k-3}, y^{k}+x_{1} y^{k-2}+\cdots+x_{k-2} y,\right. \\
& \left.x_{k-1} y^{k-1}+\cdots+x_{2 k-3} y\right),
\end{aligned}
$$

for some natural number $k$.

Example 2.7. If $n=2$ then the only stable non-immersion of corank 1 is the Whitney umbrella.

Theorem 2.8. Suppose that $f: X \rightarrow Y$ is a map of complex analytic spaces of dimensions $n$ and $n+1$ respectively, which is locally induced from codimension one maps that are stable and of corank 1 and have target of dimension N. Suppose that $Y$ is locally embedded in $\mathbb{C}^{N}$ by $p$ equations. Then

$$
\operatorname{rHd}\left(M_{k}(f) ; \mathbb{Q}\right) \geq N-p-k .
$$

Corollary 2.9. Suppose that $f: X \rightarrow Y$ is a dimensionally correct map of complete intersections of dimensions $n$ and $n+1$ respectively which is locally induced from codimension one maps that are stable and of corank 1. Then

$$
r H d\left(M_{k}(f)\right)=n-k+1 .
$$

Thus, $\mathbb{Q}_{M_{k}}^{\bullet}[n-k+1]$ is perverse.

Proof of Corollary. Since $Y$ is a complete intersection then $N-p=n+1$, and so by the theorem we have that $r H d\left(M_{k}(f) ; \mathbb{Q}\right) \geq n-k+1$. Since the map is dimensionally correct $\operatorname{dim}_{\mathbb{C}} M_{k}(f)=\operatorname{dim}_{\mathbb{C}} D^{k}(f)=n-k+1$, and so $r H d\left(M_{k}(f) ; \mathbb{Q}\right)$ is maximal. The perversity statement is then trivial.

Remark 2.10. It would seem likely that the statement of Theorem 2.8 in terms of rhd should be true, however it is not obvious how one could prove this.

\section{Proofs of the theorems}

Proof of Theorem 2.3. The assertion comes down to local considerations, and so we can assume that we have a map $F: Z \rightarrow \mathbb{C}^{n+1}$ with $F$ of degree one and $Z$ Gorenstein.

Since $Z$ is Gorenstein, by Propostions 2.5 and 3.4 of [MP] there exists a symmetric matrix, the determinant of which defines the hypersurface $F(Z)$, and the maximal minors of the matrix obtained by deleting the first column of the symmetric matrix define $M_{2}(F)$ as a complex analytic space. By Theorem 2.1 of [V] such a space is set theoretically defined by two equations. Since $M_{2}(f)=Y \cap M_{2}(F)$, we deduce from Theorem 3.2.1 of [HL] that $r h d\left(M_{2}(f)\right) \geq r h d(Y)-2$. 
The space $D_{1}^{2}(F)$ has the same locus as the conductor ideal of the map, and since $Z$ is Gorenstein the conductor ideal is principal; see Proposition 3.5 of [MP]. This implies that $D_{1}^{2}(f)$ is defined locally as a hypersurface in $X$. Hence by Theorem 3.2 .1 of $[\mathrm{HL}]$ we have $\operatorname{rhd}\left(D_{1}^{2}(f)\right) \geq \operatorname{rhd}(X)-1$.

The homology statements involving $r H d$ work because the homology statement of Theorem 3.2.1 of [HL] is also true.

To prove the first perversity theorem we sandwich the $r H d$ between the dimension of the space as follows:

$$
\operatorname{dim}_{\mathbb{C}} Y-2=r H d(Y)-2 \leq r H d\left(M_{2}(f)\right) \leq \operatorname{dim}_{\mathbb{C}} M_{2}(f)=\operatorname{dim}_{\mathbb{C}} Y-2 .
$$

The first inequality is by the homology part of the theorem, and the final equality follows from the fact that $f$ is a dimensionally correct map.

The statement for $D_{1}^{2}$ is proved by a similar argument.

The last two theorems of the previous section are in essence both consequences of the following lemma.

Lemma 3.1. Suppose that $g: W \rightarrow Z$ is a finite and proper complex analytic map and $m$ a natural number such that

$$
\operatorname{raHd}\left(D^{k}(g) ; \mathbb{Q}\right) \geq m-k \text { for } k \geq 2 .
$$

Then $r H d(g(W) ; \mathbb{Q}) \geq \min \{r H d(W ; \mathbb{Q}), m-2\}$.

Proof. Since $g$ is a complex analytic map it is possible to make $g$ a stratified submersion, so that the spaces $M_{k}(g)$ are union of strata. The finiteness of the map implies that $g$ is a Thom map.

The statement of the theorem is in essence local, so we can assume that for any point $z$ there exists a complex analytic map $F: U \rightarrow V$, where $U$ is a disjoint union of open sets in $\mathbb{C}^{N}$ and $V$ is an open set in $\mathbb{C}^{P}$ for some $N$ and $P$, and that $F \mid W \cap U=g$. We assume that locally $g(W)$ embeds in $V$ and that each disjoint set of $U$ contains one connected neighbourhood of $g^{-1}(z)$.

Let $A$ be the stratum of $g(W)$ that contains $z$. There exists a manifold $N$ in $V$ such that $N$ is transverse to the strata of $g(W)$ and $N \cap A=\{z\}$. Let the pair $\left(B_{\epsilon}, S_{\epsilon}\right) \subset N$ define the real link of the stratum $A$, i.e. $\epsilon$ is sufficiently small so that $S_{\epsilon}$, the boundary of the ball $B_{\epsilon}$ of radius $\epsilon$ centred at $z$, is transverse to the strata of $g(W)$.

Then, by definition, we have $r H d(g(W)) \geq r$ if and only if

$$
H_{i}\left(B_{\epsilon} \cap g(W), S_{\epsilon} \cap g(W)\right)=0 \text { for } i \leq r-\operatorname{dim}_{\mathbb{C}} A \text {, for all } z \in g(W) .
$$

The pair $g^{-1}\left(B_{\epsilon}, S_{\epsilon}\right)$ is a union of pairs of neighbourhoods and links of strata of the points $g^{-1}(z)$, and we have $r H d(W) \geq r$ if and only if

$$
H_{i}\left(g^{-1}\left(B_{\epsilon} \cap g(W)\right), g^{-1}\left(S_{\epsilon} \cap g(W)\right)\right)=0
$$

for $i \leq r-\operatorname{dim}_{\mathbb{C}} A$, for all $z \in g(W)$.

We can reduce the situation to the case of a zero dimensional stratum by defining the map $g^{\prime}=g \mid g^{-1}(N)$. Then the map $\varepsilon^{k}: D^{k}(f) \rightarrow Z$ provides us with an $S_{k^{-}}$-invariant set $\left(\varepsilon^{k}\right)^{-1}(N)$ defined by $\operatorname{dim}_{\mathbb{C}} A S_{k}$-invariant equations. We have $D^{k}\left(g^{\prime}\right)=D^{k}(g) \cap\left(\varepsilon^{k}\right)^{-1}(N)$. By using the alternating homology version of Theorem 3.2.1 of [HL] we deduce that

$$
\begin{aligned}
\operatorname{raHd}\left(D^{k}\left(g^{\prime}\right)\right)=\operatorname{raHd}\left(D^{k}(g) \cap\left(\varepsilon^{k}\right)^{-1}(N)\right) & \geq \operatorname{raHd}\left(D^{k}(g)\right)-\operatorname{dim}_{\mathbb{C}} A \\
& =m-k-\operatorname{dim}_{\mathbb{C}} A \text { for } k \geq 2 .
\end{aligned}
$$


Define the maps $G=g^{\prime} \mid\left(g^{\prime}\right)^{-1}\left(B_{\epsilon}\right)$ and $\tilde{G}=g^{\prime} \mid\left(g^{\prime}\right)^{-1}\left(S_{\epsilon}\right)$. Then the set $B_{\epsilon} \cap$ $g(W)$ is the image of $G$ and $S_{\epsilon} \cap g(W)$ is the image of $\tilde{G}$. The image computing spectral sequences for these maps (denoted $E(G)$ and $E(\tilde{G})$ respectively) can now be compared because the raHd of each multiple point space of $g^{\prime}$ is assumed to be known. This will then give us information on the homology of the links of $g(W)$.

The space $B_{\epsilon} \cap g(W)$ is contractible to $y$ by reducing the value of $\epsilon$, and this leads to a contraction of the multiple point spaces of $G$ to a finite set of points. Thus

$$
E_{1}^{p, q}(G)=\operatorname{AltH^{q}}\left(D^{p+1}(G)\right)=0 \text { for } q>0,
$$

and the sequence must then collapse at $E_{2}$, revealing that

$$
E_{2}^{p, 0}(G) \cong H^{p}\left(B_{\epsilon} \cap g(W)\right)= \begin{cases}0 & \text { for } p>0 \\ \mathbb{Q} & \text { for } p=0 .\end{cases}
$$

The multiple point spaces $D^{k}(G)$ and $D^{k}(\tilde{G})$ are $S_{k}$-homotopy equivalent to the union of pairs of neighbourhoods and links of the points in $\left(\varepsilon^{k}\right)^{-1}(y)$. Thus the raHd conditions on $g^{\prime}$ allow us to compare $E_{1}(\tilde{G})$ with $E_{1}(G)$. Since

$$
\operatorname{raHd}\left(D^{k}\left(g^{\prime}\right)\right) \geq m-k-\operatorname{dim}_{\mathbb{C}} A \text { for } k \geq 2,
$$

we have by definition

$$
\operatorname{Alt}^{i}\left(D^{k}(G), D^{k}(\tilde{G})\right)=0 \text { for } i<m-k-\operatorname{dim}_{\mathbb{C}} A \text { for } k \geq 2 .
$$

This implies

$$
E_{1}^{k-1, i}(\tilde{G})=\operatorname{AltH}^{i}\left(D^{k}(\tilde{G})\right)=0 \text { for } 0<i<m-k-\operatorname{dim}_{\mathbb{C}} A-1 \text { for } k \geq 2 .
$$

In a similar way to the proof of Lemma 3.11 of [Ho1] the row $E_{1}^{p, 0}(\tilde{G})$ compares to $E_{1}^{p, 0}(G)$ : The natural map $E_{1}^{p, 0}(G) \rightarrow E_{2}^{p, 0}(\tilde{G})$ is an isomorphism if $\operatorname{AltH}^{i}\left(D^{p+1}(G), D^{p+1}(\tilde{G})\right)=0$ for $i<2$. Since $E_{2}^{p, 0}(G)=0$ for $p>0$, this implies that $E_{2}^{p, 0}(\tilde{G})=0$ for $0<p<m-\operatorname{dim}_{\mathbb{C}} A-2$.

We deduce that $E_{2}^{p, q}(\tilde{G})=0$ for $p>0$ and $p+q<m-\operatorname{dim}_{\mathbb{C}} A-2, q>0$. So we have

$$
H^{i}\left(S_{\epsilon} \cap g(W)\right) \cong E_{\infty}^{0, i}(\tilde{G})=E_{1}^{0, i}(\tilde{G})=H^{i}\left((\tilde{G})^{-1}\left(S_{\epsilon}\right)\right)
$$

for $0<i<m-\operatorname{dim}_{\mathbb{C}} A-3$.

We thus deduce that

$$
H^{i}\left(G^{-1}\left(B_{\epsilon}\right),(\tilde{G})^{-1}\left(S_{\epsilon}\right)\right)=0
$$

if and only if

$$
H^{i}\left(B_{\epsilon} \cap g(W), S_{\epsilon} \cap g(W)\right)=0
$$

for $i<m-\operatorname{dim}_{\mathbb{C}} A-2$.

The former measures $r H d(W ; \mathbb{Q})$ as we noted earlier, and so we finally conclude that $r H d(g(W)) \geq \min \{r H d(W), m-2\}$.

Proof of Theorem 2.6. We calculate $\operatorname{raHd}\left(D^{k}(f)\right)$ using Proposition 3.4 of [Ho2]; from it we deduce that

$$
\begin{aligned}
\operatorname{raHd}\left(D^{k}(f)\right) & \geq k \cdot r H d(X)-(n+1)(k-1) \\
& =n-k+1 .
\end{aligned}
$$

Consider the map $g: D_{1}^{2}(f) \rightarrow Y$ given by the restriction of $f$ to $D_{1}^{2}(f)$. For this map we have $D^{k}(g)=D^{k}(f)$ for $k \geq 2$, and so by the above we have estimated 
$\operatorname{raHd}(g)$ for $k \geq 2$. From Lemma 3.1 we deduce that if $r H d\left(D_{1}^{2}(f)\right) \leq n-1$, then $r H d\left(M_{2}(f)\right) \geq r H d\left(D_{1}^{2}(f)\right)$. Since $\operatorname{dim}_{\mathbb{C}} D_{1}^{2}(f) \leq n-1$, the result is true.

The perversity result is a simple consequence of the main part of the theorem.

Theorem 2.8 is in fact deduced from the statement of the theorem for the image multiple point spaces of stable corank 1 maps.

Lemma 3.2. Suppose that $f: X \rightarrow Y$ is a stable map with only corank 1 singularities and that $X$ and $Y$ are complex analytic manifolds of dimension $n$ and $n+1$ respectively. Then,

$$
\operatorname{rHd}\left(M_{k}(f) ; \mathbb{Q}\right)=\operatorname{dim}_{\mathbb{C}} M_{k}(f)=n-k+1 .
$$

Thus $\mathbb{Q}_{M_{k}(f)}[n-k+1]$ is a perverse sheaf.

Proof. The proof is similar to the proof of Theorem 2.8 of $[\mathrm{GM}]$, where they are concerned with the simpler situation of stabilisations of mono-germ mappings.

Let Lemma $A_{r}$ be the statement that the Lemma is true for all $1 \leq k \leq r$.

Lemma $A_{1}$ is evidently true since $M_{1}$ is the image of $f$, which is a hypersurface, and so we have the stronger conclusion that $\operatorname{rhd}\left(M_{1}(f)\right)=\operatorname{rhd}(f(X))=$ $\operatorname{dim}_{\mathbb{C}} f(X)=n$.

Proof that $A_{r-1}$ implies $A_{r}$ for $r \geq 2$. Let $g=f \mid D_{1}^{r}$; then $D^{j}(g)=D_{j}^{r}(f)$ for $j<r$ and $D^{j}(g)=D^{j}(f)$ for $j \geq r$.

The map $\varepsilon^{j+1}: D^{j+1} \rightarrow D^{j}$ is a stable map between manifolds of dimension $n-j$ and $n-j+1$ respectively, and $D_{j}^{r}(f)=M_{r-j}\left(\varepsilon^{j+1}: D^{j+1}(f) \rightarrow D^{j}(f)\right.$ ) (see [GM], Theorem 2.8). So by the induction hypothesis, for $1 \leq j \leq r-1$,

$$
\begin{aligned}
r H d\left(M_{r-j}\left(\varepsilon^{j+1}: D^{j+1}(f) \rightarrow D^{j}(f)\right)\right) & =\operatorname{dim}_{\mathbb{C}} D^{j+1}(f)-(r-j)+1 \\
& =n-r+1 .
\end{aligned}
$$

The alternating rational homology groups are subgroups of the ordinary rational homology groups, so we have $\operatorname{raHd}(Z ; \mathbb{Q}) \geq \operatorname{rHd}(Z ; \mathbb{Q})$ for any $S_{k}$-invariant space $Z$. This implies that $\operatorname{raHd}\left(M_{r-j}\left(\varepsilon^{j+1}: D^{j+1}(f) \rightarrow D^{j}(f)\right)\right) \geq n-r+1$, for $j<r$.

Similarly, for $j \geq r$ the fact that $D^{j}(g)=D^{j}(f)$ is a manifold of dimension $n-j+1$ implies that $\operatorname{raHd}\left(D^{j}(g)\right)=n-j+1$.

These two estimates of $r a H d$ give that $\operatorname{raHd}\left(D^{j}(g)\right) \geq n-r-j+3$ for all $j \geq 2$. As $r H d\left(D_{1}^{r}(f)\right)=n-r+1$, we deduce from Lemma 3.1 that $r H d\left(M_{r}(f)\right) \geq$ $r H d\left(D_{1}^{r}(f)\right)=n-r+1$.

The map $f$ is stable and hence dimensionally correct, so

$$
\operatorname{dim}_{\mathbb{C}} M_{r}(f)=\operatorname{dim}_{\mathbb{C}} D^{r}(f)=n-r+1,
$$

which forces the equality of the statement.

Proof of Theorem 2.8. We again consider the local situation. By definition of $f$ there exists a stable corank 1 map $F: U \rightarrow V$, where $U$ is a disjoint union of open sets in $\mathbb{C}^{N-1}$ for some $N$, and $V$ is an open subset of $\mathbb{C}^{N}$.

Since $F^{-1}(Y)=X$, it is true that $M_{k}(f)=M_{k}(F) \cap Y$, and $Y$ is defined in $M_{k}(F)$ by $p$ equations. Lemma 3.2 states that $r H d\left(M_{k}(F)\right)=(N-1)-k+1$, and, using the rational homology version of Theorem 3.2.1 of [HL], we deduce that

$$
\begin{aligned}
r H d\left(M_{k}(f)\right) & \geq r H d\left(M_{k}(F)\right)-p \\
& =N-p-k .
\end{aligned}
$$




\section{Applichtions}

In addition to providing new examples of perverse sheaves we have the following consequences of the theorems.

Example 4.1 (cf. Example 5.2 of [Ho2]). Suppose $f: X \rightarrow Y \subset \mathbb{C P}^{n+1}$ is a finite map of complex analytic spaces locally induced from codimension one maps of degree 1 with Gorenstein domain. Then

$$
\pi_{i}\left(\mathbb{C P}^{n+1}, M_{2}(f)\right)=0 \text { for } i \leq 2 \operatorname{rhd}(Y)-n-4 .
$$

This is true because $\operatorname{rhd}\left(M_{2}(f)\right) \geq \operatorname{rhd}(Y)-2$ by Theorem 2.3(i), and then we apply Theorem 4.4 of [Ho2]. This improves the estimate of Example 5.2 of [Ho2].

Example 4.2. Suppose that $X$ is a complex analytic manifold of dimension $n$ and that $f: X \rightarrow \mathbb{C P}^{n+1}$ is a complex analytic map with finitely $\mathcal{A}$-determined corank 1 singularities. Then,

$$
H_{i}\left(\mathbb{C P}^{n+1}, M_{k}(f) ; \mathbb{Q}\right)=0 \text { for } i \leq n-2 k+2 .
$$

This is because by Example 2.2 such a map is locally induced from a stable corank 1 map, and so by Corollary 2.9 we have that $r H d\left(M_{k}(f)\right)=n-k+1$. The equality comes from $f$ being dimensionally correct. Theorem 4.6 in [Ho2] provides the global conclusion.

With Theorem 2.8 we can improve Theorem 2.8 of [GM] to a theorem involving the stabilisation of multi-germ mappings and not just mono-germs.

Corollary 4.3 (cf. [GM, Theorem 2.8]). Suppose $f: X \rightarrow Y$ is a stable perturbation of a finitely $\mathcal{A}$-determined corank 1 map multi-germ $f_{0}:\left(\mathbb{C}^{n}, S\right) \rightarrow\left(\mathbb{C}^{n+1}, 0\right)$ for some finite set $S$. Then the spaces $M_{k}(f)$ have reduced rational homology only in dimension $n-k+1$.

Proof. The map $f_{0}$ can be unfolded by a map $F:\left(\mathbb{C}^{n} \times \mathbb{C}, S \times 0\right) \rightarrow\left(\mathbb{C}^{n+1} \times \mathbb{C}, 0 \times 0\right)$ so that $f_{t}(x):=F(x, t)$ is such that $f_{t}$ is stable for $t \neq 0$ and small. Such a map $f_{t}$ is a stabilisation of $f_{0}$.

The map $F$ is induced from a codimension 1 map with stable corank 1 singulari-

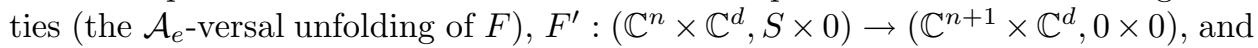
the image of $F$ is defined in the image of $F^{\prime}$ by $d-1$ equations. Thus by Theorem 2.8 we have

$$
r H d\left(M_{k}(F) ; \mathbb{Q}\right) \geq n-k+2 .
$$

Because of the dimension of $M_{k}(F)$ this is in fact an equality. The maps $\pi_{k}$ : $M_{k}(F) \rightarrow \mathbb{C}$ induced from the obvious projection $\pi^{\prime}: \mathbb{C}^{n+1} \times \mathbb{C} \rightarrow \mathbb{C}$ have isolated singularities at $(0,0)$ in the sense of Lê; this is proved in the same way as in $[\mathrm{M}]$. Hence, by Corollary 5.5 of [L], the fibres have reduced rational homology only in dimension $n-k+1$. The fibres of the map are $M_{k}\left(f_{t}\right)$, and so the corollary is proved.

In fact, using Theorem 2.3(i) we can prove that in the above situation the space $M_{2}(f)$ is a wedge of $(n-1)$-spheres. From experience, it seems natural to conjecture that all the spaces $M_{k}$ above are homotopically equivalent to wedges of spheres. This would be true if the conclusion to Theorem 2.8 could be proved for $r h d$ and not just rational rectified homological depth. 


\section{REFERENCES}

[GM] V.V. Goryunov and D. Mond, Vanishing cohomology of singularities of mappings, Compositio Math., 89 (1993), 45-80. MR 94k:32058

[HL] H. Hamm and Lê Dũng Tráng, Rectified homotopical depth and Grothendieck Conjectures, in The Grothendieck Festschrift, Volume II, Progress in Mathematics 87, (Birkhäuser, Boston 1990), 311-351. MR 92j:32131

[Ho1] K. Houston, Local Topology of Images of Finite Complex Analytic Maps, Topology 36 (1997), 1077-1121. CMP 97:11

[Ho2] K. Houston, Global Topology of Images of Finite Complex Analytic Maps, to appear in the Mathematical Proceedings of the Cambridge Philosophical Society.

[L] Lê Dũng Tráng, Complex analytic functions with isolated singularities, J. Algebraic Geometry, 1 (1992), 83-100. MR 92j:32132

[M] R. MacPherson, Global questions in the topology of singular spaces, Proceedings of the International Congress of Mathematicians, Warsaw 1983, pp. 213-235. MR 86m:58016

[M] W.L. Marar, Mapping Fibrations, Manuscripta Mathematica, 80 (1993), 273-282. MR 94i:32058

$[\mathrm{MM}]$ W.L. Marar and D. Mond, Multiple point schemes for corank 1 maps, J. London Math. Soc. (2)39 (1989) 553-567. MR 91c:58010

[MP] D. Mond and R. Pellikaan, Fitting ideals and multiple points of analytic mappings, in Algebraic Geometry and Complex Analysis, ed. E. Ramirez de Arellano, Springer Lecture Notes in Mathematics 1414, Springer-Verlag, Berlin, 1990, 107-161. MR 91e:32035

[V] G. Valla, On determinantal ideals which are set-theoretic complete intersections, Compositio Mathematica, Vol 42 (1981), 3-11. MR 83g:14027

[W] C.T.C Wall, Finite determinacy of smooth map-germs, Bull. London Math. Soc., 13 (1981), 481-539. MR 83i:58020

School of Mathematics and Statistics, Middlesex University, Bounds Green Road, London, N11 2NQ, United Kingdom

E-mail address: k.houston@mdx.ac.uk 